\title{
Assessment of Focal Hot Spot in Right Supra-orbital Region Characterized with 99m Tc-MDP SPECT-CT A Case Report
}

Puja Bhattacharjee, Jasmine Ara Haque, Simoon Salekin, Tapati Mandal, Sadia Salam, Sharmin Reza, Sharmin Quddus, Mostofa Shamim Ahsan and Abu Bakker Siddique

\author{
National Institute of Nuclear Medicine and Allied Sciences (NINMAS), Dhaka, Bangladesh
}

Address of Correspondence: Puja Bhattacharjee, Medical Officer, Scintigraphy Division, National Institute of Nuclear Medicine and Allied Sciences (NINMAS), BSMMU, Dhaka.email:pujadmc68@gmail.com

\begin{abstract}
Lesion at the superior orbital margin is infrequently seen on MDP bone scintigraphy, however the exactetiology cannot always be clearly explained. Here, a case is presented showing focal uptake of MDP tracer in right supra-orbital region. Single photon emission computed tomography/computed tomography (SPECT/CT) was done to localize and characterize this undetermined lesion.

Keywords: Bone scan, right supra-orbital region, SPECT/CT

Bangladesh J. Nucl. Med. Vol. 22 No. 1 January 2019

Doi : https://doi.org/10.3329/bjnm.v22i1.40516
\end{abstract}

\section{INTRODUCTION}

Bone scintigraphy is a useful tool for the detection of bone metastasis as well as staging of cancer patient. Technetium-99m methylene diphosphonate $(99 \mathrm{mTc}$ MDP) is a tracer of choice to detect the site of metastasis. This method is an easy \& less time consuming procedure for the detection of bony involvement in cancer staging. Bone scintigraphy is also used to determine inflammatory \& degenerative conditions. Many latent focal activity demonstrating such conditions are found during routine bone scintigraphy; whereas the usual aim was to differentiate between metastatic \& non-metastatic stage (1).Thus incidental finding of this type of focal hot spot may represent synchronous primary origin of new lesion or lesion of benign origin. In this report a patient of prostatic malignancy undergoing bone scintigraphy who demonstrated an incidental focal uptake at right supraorbital region during whole body scintigraphy, which wasfurther evaluated using single photon emission computed tomography/ computed tomography (SPECT-CT) to localize and characterize the lesion.

\section{CASE REPORT}

A patient of 65 years old male, diagnosed as a case adeno carcinoma of prostate; Gleason's score $4+3=7$. He underwent TURP for resection of the growth. Following TURP his MRI showed persistent malignant growth in prostate with extra-prostatic extension to the urinary bladder \& sigmoid colon. For this he was given three doses of chemotherapy. His recent PSA was $>400$ $\mathrm{ng} / \mathrm{mol}$. Then he was presented to our scintigraphy department at NINMAS to determine the presence of any bone metastasis following chemotherapy.

Following administration of $20 \mathrm{~m} \mathrm{Ci}$ of $99 \mathrm{~m} \mathrm{Tc}$ MDP, delayed whole body bone imaging was done at 03 hours post injection. The result demonstrated multiple focal areas of increased radiotracer concentrations over superior lateral margin of the right orbit, upper part of both humerus and right acetabular region on the anterior view. Tracer concentrations in the humerus \& right acetabular region were consistent with skeletal metastases; however the location of the tracer concentration in the right supraorbital region was not clearly defined (Figure 1). Thereby additional SPECT-CT correlation was necessary. SPECTCT correlation of right supra orbital region was done and result demonstrated a rounded well circumscribed tracer accumulation in the right supra orbital area without any ophthalmic tissue involvement; consistent with secondary metastasis (Figure 2). CT scan revealed no abnormality. 


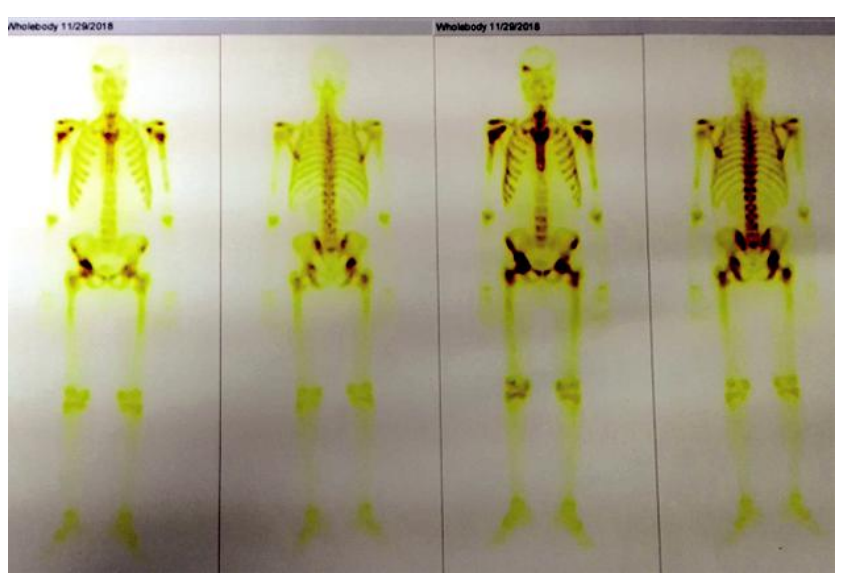

Figure 1: $99 \mathrm{mTc}$ whole body bone scan of prostate carcinoma showing focal tracer uptake at right supra-orbital margin, both humeri and right acetabular region.

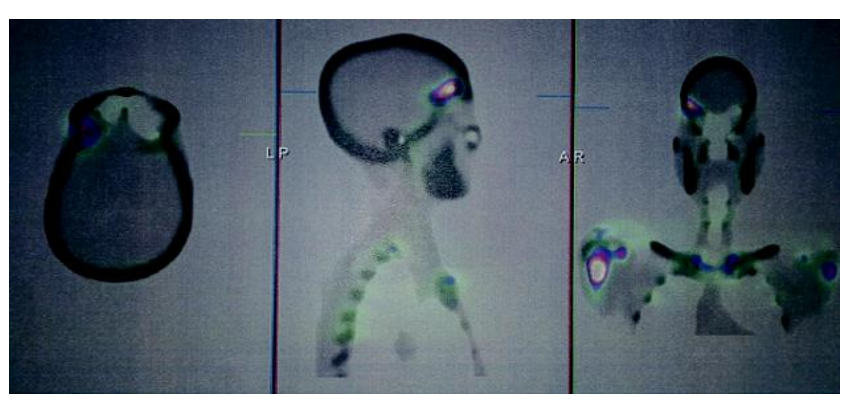

Figure 2: SPECT-CT of a prostate carcinoma patient demonstrates tracer uptake in right supraorbital margin.

\section{DISCUSSION}

Bone scan is one of the most common nuclear medicine procedures, mostly used for the evaluation of benign bone disease like infection/inflammation and also in diagnosing metastatic spread of disease (1). Sensitivity of bone scan is higher though its specificity is lower in benign conditions. Widespread involvement usually suggests metastases in known primary cases; however metabolic disease always needs to be differentiated. Solitary lesion is almost always a difficult to diagnose. In Those cases SPECT-CT correlation may give additional information.

Bone scan is highly sensitive to detect bone lesions, but specificity is limited in characterizing lesions (2). However, specificity is mostly limited to differentiate between malignant $\&$ benign lesions. In patients with solitary bone lesions with extra osseous malignancies, comprises approximately one-half in extra-articular regions are found to be metastatic comparing to approximately $60-70 \%$ for in the vertebra or pelvis, and about $80 \%$ for sternal lesions in patients with breast cancer $(3,4)$.

For focal lesion in the calvareum approximately $20 \%$ of such lesions in patients with underlying extraosseous tumors appear to be metastatic. However, many focal areas remain inconsistent. One such pattern of uptake is infrequently seen at the superior lateral margin of the orbit. Differential diagnosis of this type uptake may be benign bony lesions like fibrous dysplasias, osteomas, enchondromas, hemangiomas, meningiomas, posttraumatic changes and extra-osseous uptake in the lacrimal gland or malignant lesion of newer primary or even metastasis from second primary lesion.

Hybrid SPECT/CT imaging in this patient was analyzed for further characterizing and localizing this commonly seen pattern of focal calvarial uptake at the supero lateral margin of the orbit.

Focal lesion in the calvarium are significantly better displayed with SPECT-CT fusion than planar bone scintigraphy or CT alone differentiating benign and malignant lesions. SPECT fused with CT significantly increases value for making a specific diagnosis. Often patients with malignant tumors have non-specific symptom. In these cases, it is important to rule out metastasis. The cause of benign focal uptake should be evaluated carefully because many underlying diseases that can influence treatment. In the interpretation of bone scans, it is important to recognize benign patterns of tracer uptake to avoid overstatement of bone metastasis. The pattern of tracer uptake at the superior lateral margin of the orbits on planar bone scintigraphy is infrequent but localizing \& characterizing this type uptake pattern is non-specific.

\section{CONCLUSION}

In this case, similar tracer uptake in supraorbital margin to other suggested metastatic bony lesions demonstrate the efficacy of hybrid SPECT-CT imaging. 


\section{REFERENCES}

1. Hamaoka T, Madewell JE, Podoloff DA, Hortobagyi GN, Ueno NT. Bone imaging in metastatic breast cancer. J Clin Oncol 2004;22:2942-53. DOI: 10.3238/arztebl.2014.0741

2. Schillaci O, Danieli R, Manni C, Simonetti G. SPECT/CT with a hybrid camera useful to improve scintigraphic imaging interpretation. J Nucl Med Commun 2004; 25:705-710 6.DOI: 190: 1085-1090. 10.2214/AJR.07.2107

3. Utsunomiya D, Shiraishi S, Imuta M, et al. Added value of SPECT/CT fusion in assessing suspected bone metastasis: comparison with scintigraphy alone and nonfused scintigraphy and CT. Radiology 2006;238:264-271 PMID: 16304081 DOI:10.1148/radiol.2373041358

4. Klaus Strobel1, Cyrill Burger1, Burkhardt Seifert2, Daniela B. Husarik1, Characterization of Focal Bone Lesions in the Axial Skeleton: Performance of Planar Bone Scintigraphy Compared with SPECT and SPECT Fused with CT. Americal J of Roent 2007;188(5):W467-W474. DOI: 10.2214/AJR.06.1215. 